\title{
THYMIC HYPERPLASIA IN GRAVES' DISEA
WAIT AND SEE, OR INTERVENE?
}

\author{
KAMATH C (1), MCALEER B (2), ADLAN MA (2), PREMAWARDHANA LD (1,2)
}

\begin{abstract}
Centre for Endocrine and Diabetes Sciences, University Hospital of Wales (1), Heath Park, Cardiff, CF14 4XN Section of Endocrinology, YYF Hospital (2), Ystrad Fawr Way, Caerphilly CF82 7EP, UK
\end{abstract}

\section{Introduction and Case Presentation}

Thymic hyperplasia (TH) (figures 1,2) occurs in autoimmune endocrine diseases such as Graves' disease (GD) and Addison's disease (AD) and is possibly related to its role in autoimmunity. Its true incidence is not known as thymic imaging is not undertaken routinely, and TH is usually diagnosed as a "thymic incidentaloma" in these conditions.

We present 3 subjects with TH complicating GD and $A D$, who were managed expectantly, without intervention.

\section{Subject 1}

Subject 2

Subject 3

Clinical presentation

37 year old female

Osmotic symptoms ++, Weight loss - 5 stones

Pulse 120, BP 121/81 lying; 99/70 standing

Goitre, smooth, symmetrical, bruit +, No pigmentation

Investigations, Diagnosis and Progress

(a) free T3 > 46.1; free T4 - 59.5; TSH $<0.01$

(b) Thyrotrophin Receptor Ab (TRAb) - 25.5

(c) Corrected calcium 2.98; (d) PTH $<0.5$

(e) Short Synacthen test - cortisol 305 (0min) and 343 (30min); Adrenal antibodies - positive

(f) CT Thorax ABNORMAL (Figures 1a and 1b)
36 year old female - seen in chest clinic Breathlessness, weight loss

Referred to thoracic surgeons - CT abnormal

Referred to Thyroid clinic - thyrotoxic

(a) Free T3 - 17; free T4 32; TSH $<0.02$

(b) TRAb - 3.6 (after 7 months of treatment)

(c) CT Thorax ABNORMAL (Figures 2a and 2b)
47 year old female Breathlessness + Chest pain Diagnosis - ? PE Weight loss, "Shakiness"

\section{GD and $A D$, presenting with hypercalcaemia}

Clinically and biochemically stable on carbimazole, hydrocortisone and fludrocortisone

\section{Figure 1a}

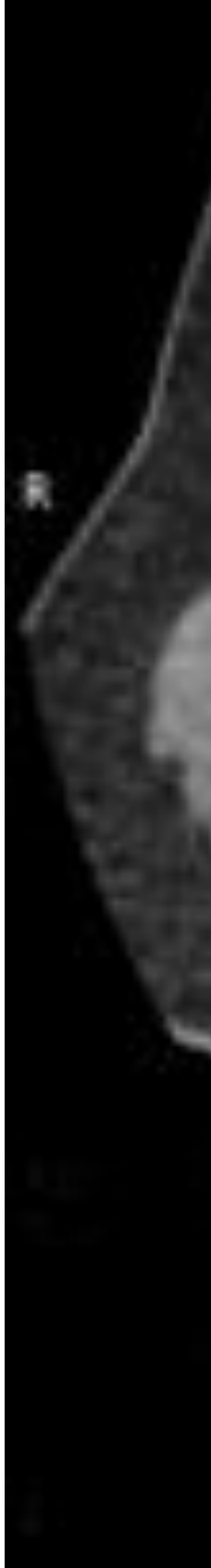

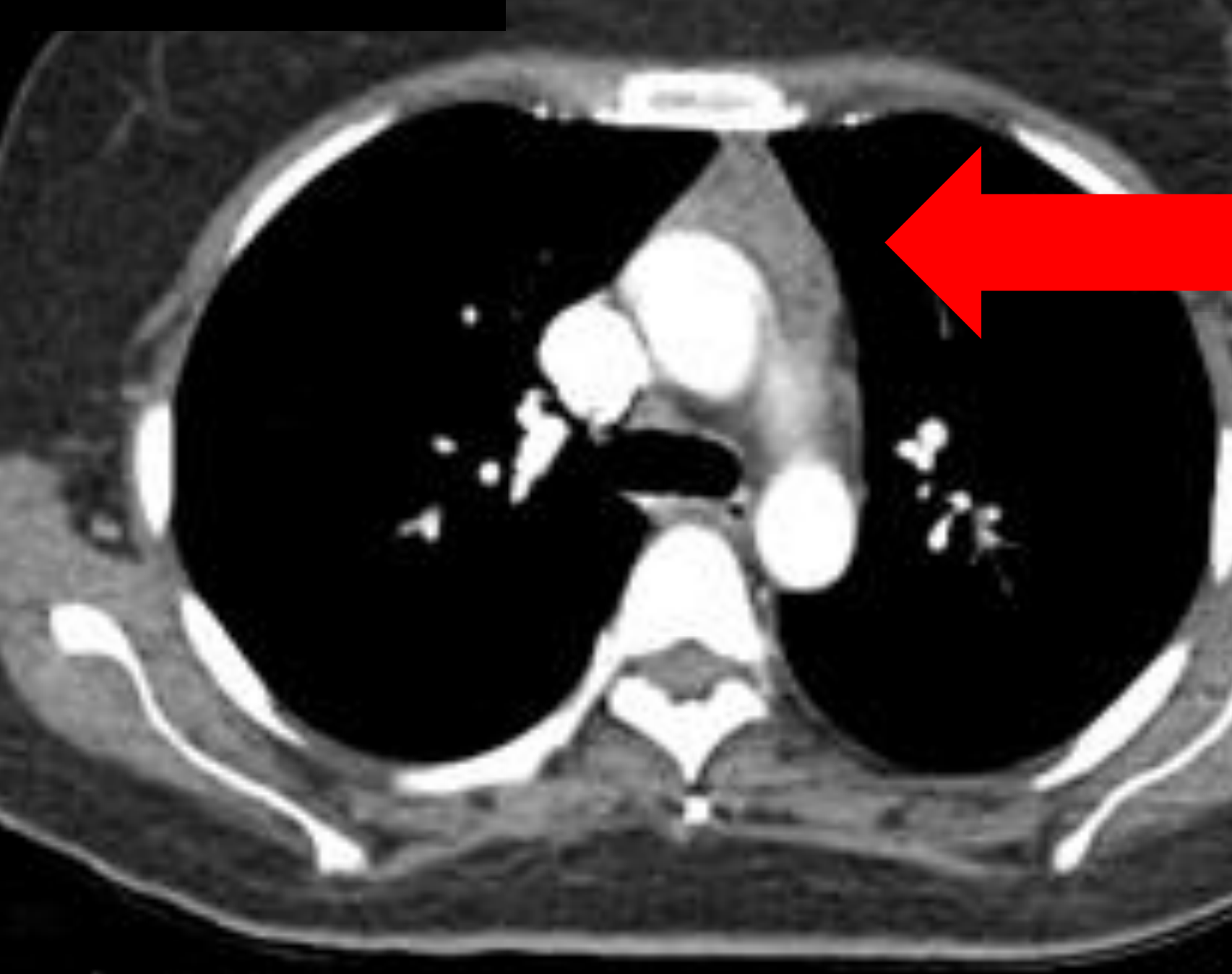

\section{Benign thymic}

hyperplasia

(red solid arrows)

1. Arrowhead appearance

2. Sharp regular margins

3. No infiltration of organs

4. Isodense with muscles

4. No cysts / calcification

BOX 1
Figure $1 \mathrm{~b}$

Figure $2 \mathrm{a}$

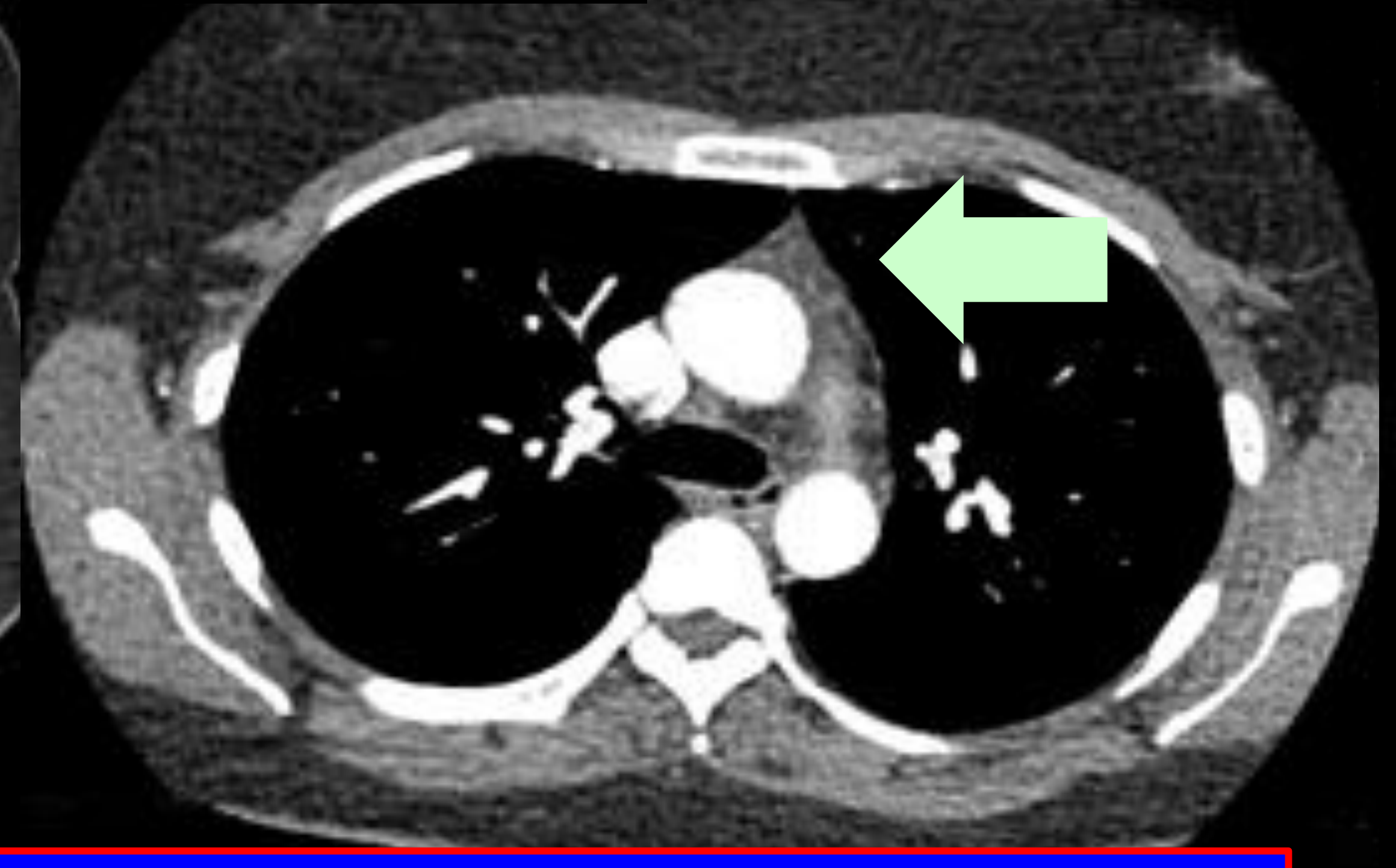

Significant regression of thymus (a) Free T3 - 6.7; free T4 18.7; $\mathrm{TSH}<0.02$

(b) TRAb - 4.5

(c) CTPA ABNORMAL (similar appearances to Fig 1a, 2a)
GD - thoracic surgery postponed

Stable on carbimazole
GD - T3 toxicosis

Stable on carbimazole

(1)

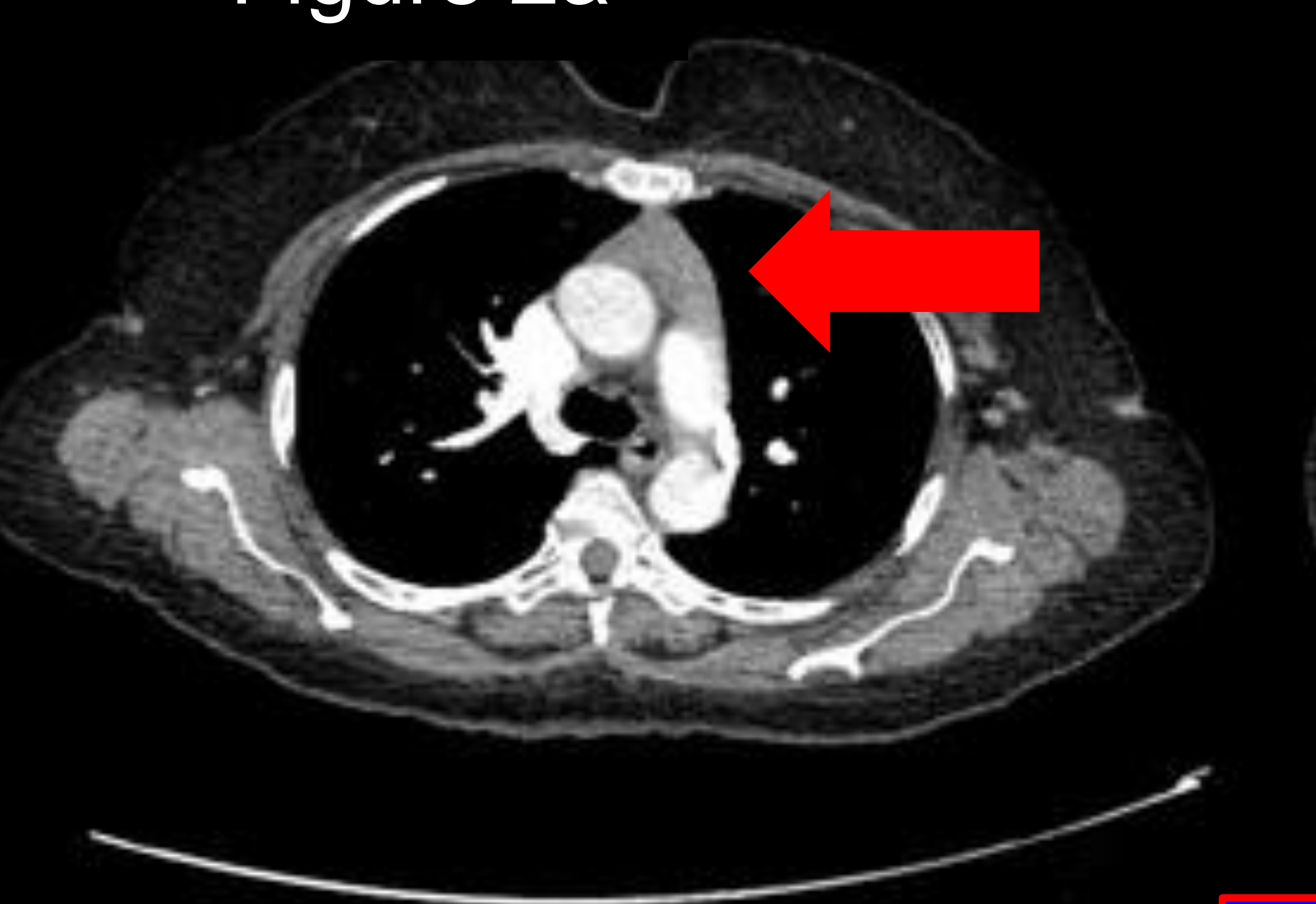

Figure 2b

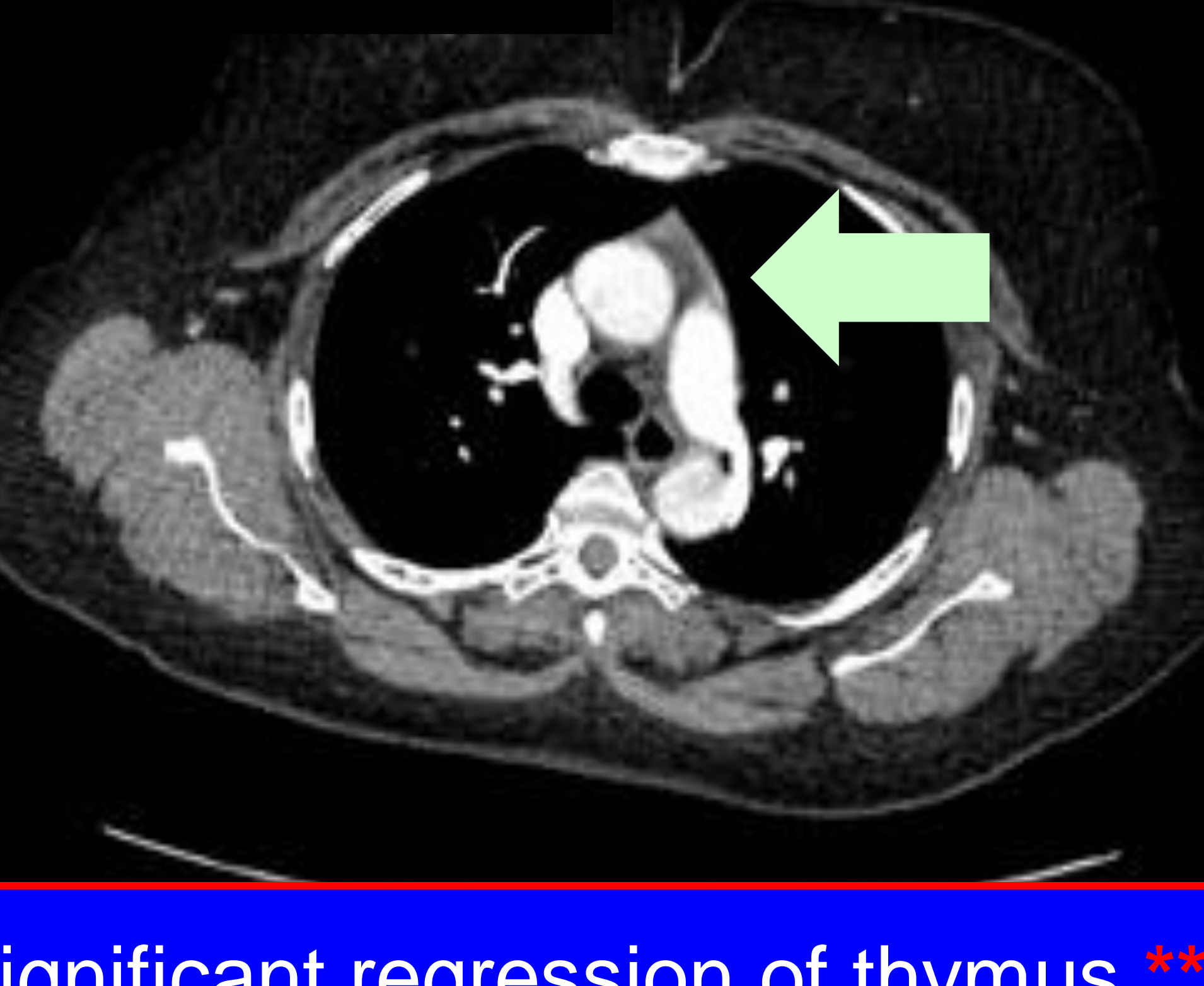

Significant regression of thymus

\section{Discussion}

(1) Thymic hyperplasia in GD, is benign in the vast majority - CT scan appearances are helpful in differentiating between benign and malignant enlargement (Box 1)

(2) There is evidence that the thymus regresses in the majority after treatment of GD

(3) Interval scanning should be done several months after control of thyrotoxicosis

(4) Two of our subjects had regression of their TH when GD was treated with thionamides (Figures $1 \mathrm{~b}$ and $2 \mathrm{~b}$ ) - the third awaits interval scanning

(5) We recommend that TH in GD should be managed expectantly with interval scans after 6 months of thionamide therapy, if initial scans confirm a benign morphology

(6) Major surgery may thus be avoided in these metabolically fragile subjects 